\title{
Design an Adaptive Quality Control Phantom to Optimize QC Test Methods
}

\author{
Hossein Zamani Zeinali ${ }^{*}, 1$, Mehdi Ghiassi-Nejad ${ }^{2}$ and Aliakbar Mirzaii ${ }^{1}$ \\ ${ }^{I}$ Department of Physics, Amirkabir University of Technology, Tehran, Iran \\ ${ }^{2}$ Department of Biophysics, Tarbiat Modares University, Tehran, Iran
}

\begin{abstract}
The main objective of this research is to introduce a newly developed device called as "Adaptive Quality Control Phantom" (AQCP) that designed to perform the QC tests. AQCP is a computer-controlled phantom which positions and moves a radioactive source in the FOV of an imaging nuclear medicine device on a definite path to produce any spatial distribution of gamma rays to simulate $\mathrm{QC}$ phantoms. To establish and prove the proper functionality as well as the accurate performance of AQCP, different tests including systematic uniformity, collimator hole angulation and the center of rotation tests have been conducted by this device and then the results, findings and differences of such testing when comparing to what achieved by the QC classic method tests have been discussed and analyzed in detail in this paper. According to the different tests done by AQCP, the authors found that the performance of systematic uniformity test shows a considerable reduction in the technologist dose compared to the IAEA-TECDOC-602 method. The collimator hole angulation for LEHR, LEUHR and LEHS collimators were measured by using a point source and the computer-controlled cylindrical positioning and the results achieved indicate that the measurement accuracy for absolute angulation errors was better than 0.018 degrees. A method for center of rotation assessment by AQCP was introduced, the results of such proposed method compared to the routine QC test and their differences have been discussed in detail. Based on all discussed in this paper regarding AQCP, the authors suggest that their presented device would be able to simulate QC phantoms.
\end{abstract}

Keywords: nuclear medicine, adaptive quality control phantom, systematic uniformity, collimator hole angulation, center of rotation.

\section{INTRODUCTION}

Single Photon Emission Computerize Tomography (SPECT) has a number of advantages over conventional Nuclear Medicine (NM) imaging: Contrast improvement and Total volume imaging [1-6]. In order to realize these advantages, rigorous QC procedures must be performed on a routine basis. Important considerations for tomography, unlike planar imaging, include flood field uniformity and Center of Rotation (COR) correction/verification and Collimator Hole Angulation (CHA) $[1,4,7,8]$.

The flood field uniformity of a scintillation camera is the ability of the camera to produce a uniform image when exposed to a homogeneous spatial distribution of gamma rays $[9,10]$. The extrinsic uniformity was assessed for any collimator using a $10 \mathrm{mCi}{ }^{99 \mathrm{~m}} \mathrm{Tc}$ flood phantom placed on any collimator. The IAEA protocol for extrinsic uniformity analyzed Uniformity over the Useful Field Of View (UFOV) with a flood image counting 1 to 3 million counts [5].

As we know, the intrinsic floods are often being used and preferred than the extrinsic ones due to its lower radiation exposure to the staff, the higher purchasing cost of flood source or phantom as well as less experiment time when acquiring the high count floods $[10,11]$. We tried to measure systematic uniformity of gamma camera SPECT with lower technologist dose by AQCP method.

For SPECT imaging, the angulation of the holes in parallel hole collimator must be known in order to ensure its

\footnotetext{
*Address correspondence to this author at the Department of Physics, Amirkabir University of Technology, Mail Box No. 14665-971, Tehran, Iran; E-mail: hzamanizeinali@yahoo.com
}

proper set up during a tomographic acquisition, i. e., its holes must be perpendicular to the Axis of Rotation $[2,4,6]$. One method for measuring CHA, which is a quantative method and is used by the authors is the displacement of the image of a point source that is examined as the source is moving vertically away from the collimator face. To apply such method, it is necessary to use a phantom like Adaptive Quality Control Phantom (AQCP) or jig to support the source at two vertical distances from the collimator face to ensure that the accurate alignment of the source is obtained $[2,4]$. We tried to measure CHA of three low energy collimators with more accuracy by AQCP method.

According to the IAEA-TECDOC-602 method for the assessment of COR, the radioactive point source will be placed at a distance $\mathrm{R}$ off the axis of rotation and a SPECT acquisition through $360^{\circ}$ around this point source will be obtained $[2,4,5]$. For COR measuring, a 360 degree tomographic study must be provided by using a $64 \times 64$ matrix, $20 \%$ window and 64 projections. Each projection would require $50 \mathrm{~K}$ counts acquisition $[4,5,8]$. In the IAEA-TECDOC602 method of COR evaluation, the constant shift of COR correction would be measured and applied [8]. We tried to measure dynamic mechanical rotation error of gamma camera SPECT by evaluating the results of COR with IAEATECDOC-602 and AQCP methods.

AQCP designed to perform a uniform set of procedures that can be used for routine quality control of a scintillation camera-based system. AQCP is an electromechanical device designed to acquire the field uniformity, center of rotation and collimator hole angulation by using some advanced methods of position control [12-14]. For using AQCP to simulate QC phantoms, the device moves a radioactive source within the Field Of View (FOV) of the NM imaging 
device on a definite path to produce a three-dimensional artificial distribution of $\gamma$ rays [12-14]. AQCP, in deed, can be used to optimize QC tests for the measurement of uniformity, CHA and COR of SPECT.

\section{MATERIALS AND METHODS}

AQCP design and structure: $\mathrm{AQCP}$ is structured of different parts comprising mechanical and electrical and is equipped with hardware and software systems. From mechanical perspective, the cylindrical coordinates is chosen as a pattern for the motion and simulation of AQCP [12,13]. A combination of ball screw and reel system are used to position the point source in any desired point in space with a precision of $0.06 \mathrm{~mm}$ (Figs. 1,2). Two step motors are used; each is connected to a gear box and divides each complete turn into 2000 steps (0.18 degree/step). The accuracy of step motor is $10 \%$ ( 0.018 degree/step). To achieve the $\mathrm{Z}$ and $\theta$ (two dimensions of the cylindrical coordinates), by the AQCP software, the ball screw system was used (Figs 1,2). Ball screw consists of a precise screw with a step of $1 \mathrm{~mm}$ and a wide nut (with length of $4 \mathrm{~cm}$ ). Another small nut was put under the wide nut. The $\theta$ parameter is adjusted by changing the position of the small nut and fixing it to the wide nut. When the small nut is open, the system can give different $Z$ 's. So, in this system the $Z$ and $\theta$ can be adjusted manually. To reach $r$ (third dimension of the cylindrical coordinates), the related motor (motor No. 1 In Fig. (1)) rotates the reel and the roll. A complete round of the reel $(30 \mathrm{~mm})$ is 500 steps, as a result; the precision of transverse radius of cylindrical coordinates (r) is $0.06 \mathrm{~mm}$. From electrical standpoint, AQCP is equipped with two driver and actuator circuits of step motors which together with other parts help the whole system function and operate properly and accurately. AQCP's overall functions are being controlled by a software interface that is developed to position the cylindrical coordinates $[13,14]$. The user of the system would be able to define and design a path for moving the radioactive source. The software draws the path on the screen and analyzes the results of the experiment. The Haematocrit-capillaries with external diameter of 1.5-1.6 $\mathrm{mm}$ and a radionuclide, i.e. ${ }^{99 \mathrm{~m}} \mathrm{Tc}$ with high specific activity (more than $50 \mathrm{mCi} / \mathrm{cc}$ ) were used to make a point or a line source. A small drop of ${ }^{99 m} \mathrm{Tc}$ with a diameter of $1 \mathrm{~mm}$ was used to make the point source and a small amount of ${ }^{99 \mathrm{~m}} \mathrm{Tc}$ was used to make the line source with a length of $4 \mathrm{~cm}$. The gamma camera SPECT system under examination was SMV double-head gamma camera model DSX-XL SPECT.

Planar system uniformity test: The planar system uniformity was assessed for the camera fitted with the Low Energy High Resolution (LEHR) collimator with an energy range of $60-140 \mathrm{keV}$ and sensitivity $235(\mathrm{cpm} / \mu \mathrm{Ci})$ as well as using a $10 \mathrm{mCi}^{99 \mathrm{~m}} \mathrm{Tc}$ flood phantom placed on the parallel hole collimator. The IAEA protocol for systematic flood

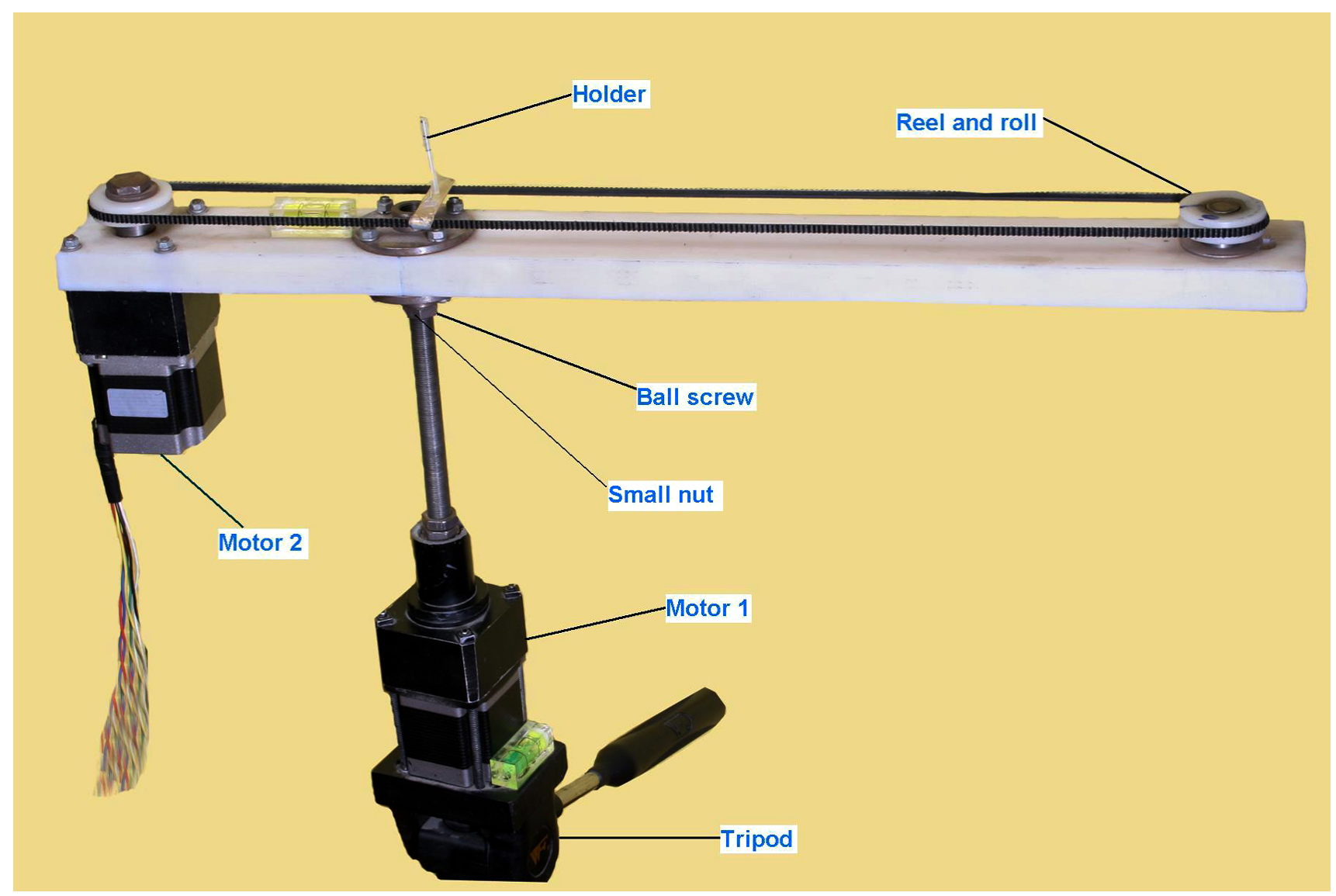

Fig. (1). AQCP mechanical section. Ball screw and Reel and roll systems were used to position the point source in any desired point in space with a precision of $0.06 \mathrm{~mm}$. To get the $\mathrm{Z}$ and $\theta$ the ball screw system was used. Ball screw consists of a precise screw with a step of $1 \mathrm{~mm}$ and with a wide nut (with length of $4 \mathrm{~cm}$ ). Another small nut was put under the wide nut. The $\theta$ parameter is adjusted by changing the position of the small nut and fixing it to the wide nut. When the small nut is open, the system can give different Z's. So, in this system the Z and $\theta$ can be adjusted manually. To get $r$ related motor rotates the reel and the roll. 


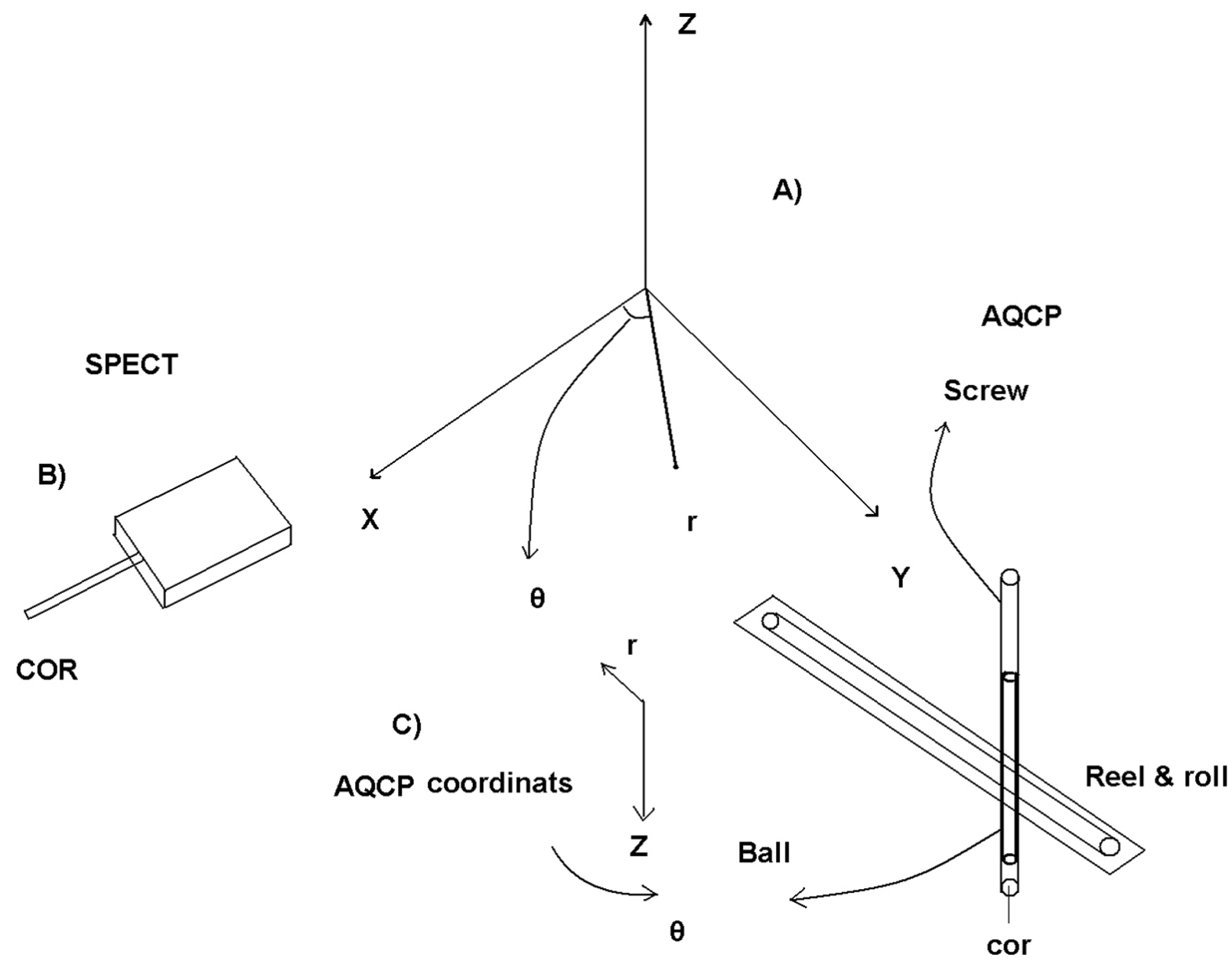

Fig. (2). Schematic drawing of a SPECT Center of Rotation (COR) and AQCP center of rotation (cor).

field uniformity analyzes Integral Uniformity (IU) over the Useful Field Of View (UFOV) [5]. The IU represents the maximum pixel count difference over the indicated field of view expressed in percentage.

Integral Uniformity of uniform images can be measured by using relation 1 given below:

$\mathrm{IU}=(\operatorname{Max}-\mathrm{Min}) /(\operatorname{Max}+\operatorname{Min}) * 100$

Here, Max and Min are Maximum and Minimum pixels count within the $\mathrm{UFOV}=0.95 * \mathrm{FOV}$, respectively.

For measuring of the planar system uniformity with AQCP, the line source is moved over the field of view in a precisely defined condition as illustrated below:

Length of the source $=4 \mathrm{~cm}$, Inner diameter of the source $\leq 1.6 \mathrm{~mm}$ activity $1 \mathrm{mCi}$, window: $20 \%$ and measuring distance source to collimator $=10 \mathrm{~cm}$.

To irradiate all the pixels in a uniformly manner, the timing and speed of the motion as well as the delay time between 2 steps of step motors should be adjusted and to meet this feature, a particular attention should be paid to the path and the velocity of the moving source. Uniformity image taken by AQCP method was evaluated by calculating IU parameter from relation No:1 same as the IAEA-TECDOC602 method.

The IU and absorbed dose of technologist have been examined while comparing with each other by the two afore- described methods. The technologist dose was measured by electronic personal dosimeter model DoseGUARD s 10 with an energy range of $50 \mathrm{keV}-3 \mathrm{Mev}$.

Collimator Hole Angulation (CHA) test: For measuring of the CHA with AQCP, the point source was positioned in 140 point within the FOV under the following defined conditions: length of the source $\leq 2 \mathrm{~mm}$, Inner diameter of the source $\leq 1.6 \mathrm{~mm}$, activity $200 \mu \mathrm{Ci}$ of ${ }^{99 \mathrm{~m}} \mathrm{Tc}$, window: $20 \%$. The CHA was evaluated for the camera fitted with 3 collimators including Low Energy High Resolution (LEHR), Low Energy High Sensitivity (LEHS) and the Low Energy Ultra High Resolution (LEUHR) collimators. The characteristics of collimators shown in Table $\mathbf{1}$.

For each of those collimators listed above, two images were taken at 140 different points of collimators with matrix size of $256 * 256$ at two vertical distances of $Z 1=10 \mathrm{~cm}$ as position 1 and $Z 2=13 \mathrm{~cm}$ as position 2 from the collimator and at each point of the collimators positions, the point source was imaged for a total of 50k counts.

The data analysis of the two images are described in position 1 and 2 are initiated with application of a stand are nine point smoothing kernel to reduce the random fluctuation in the data. The approximate $\mathrm{X}$ and $\mathrm{Y}$ coordinates (COGX and COGY) of each point source image is determined by calculation of the centroids of each point source image from relations 2 and $3[4,5]$. 
Table 1. Summary of Collimator Characteristics

\begin{tabular}{|c|c|c|c|c|c|c|}
\hline Label & Length $(\mathbf{m m})$ & Hole $(\mathbf{m m})$ & Septa $(\mathbf{m m})$ & Sensitivity $(\mathbf{C p m} / \boldsymbol{\mu C i})$ & System Resolution At 10 cm & Energy Range in keV \\
\hline \hline LEHR & 46 & 2.032 & 0.152 & 235 & $8.0 \mathrm{~mm}$ & $60-140$ \\
\hline LEUHR & 47 & 1.778 & 0.127 & 176 & $7.1 \mathrm{~mm}$ & $60-140$ \\
\hline LEHS & 47 & 2.870 & 0.203 & 460 & $10.7 \mathrm{~mm}$ & $60-140$ \\
\hline
\end{tabular}

$\mathrm{COGX}=\Sigma \mathrm{i} \mathrm{C}(\mathrm{i}, \mathrm{j}) / \Sigma \mathrm{C}(\mathrm{i}, \mathrm{j})$

$\mathrm{COGY}=\Sigma \mathrm{j} C(\mathrm{i}, \mathrm{j}) / \Sigma \mathrm{C}(\mathrm{i}, \mathrm{j})$

Here, $C(i, j)$ is count in pixel $(i, j)$. For each of the 140 points of the collimators the CHAX and CHAY were determined by relations 4 and $5[2,4,5]$.

$\mathrm{CHAX}=\operatorname{Arctan}[\mathrm{K} 1 \times \mathrm{K} 2 \times(\mathrm{COGX}(\mathrm{Z} 1)-\mathrm{COGX}(\mathrm{Z} 2))]$

$\mathrm{CHAY}=\mathrm{Arctan}[\mathrm{K} 1 \times \mathrm{K} 2 \times(\mathrm{COGY}(\mathrm{Z} 1)-\mathrm{COGY}(\mathrm{Z} 2))]$

In relation 4,5 K1= $1 /(\mathrm{Z} 1-\mathrm{Z} 2)$ and $(\mathrm{Z} 1-\mathrm{Z} 2)$ distance between two images in $\mathrm{mm}$ (for all measurements $30 \pm 0.06 \mathrm{~mm}$ ) And K2=Pixel size (mm/pixel).

The Root Mean Square (RMS) of CHA can be obtained by:

$\mathrm{CHA}=\left(\mathrm{CHAX}^{\wedge} 2+\mathrm{CHAY}^{\wedge} 2\right)^{\wedge} 0.5$

Center of Rotation Test: According to the IAEATECDOC-602 method for assessment of COR, the radioactive point source is placed at a distance $\mathrm{R}$ off the axis of rotation and a SPECT acquisition through $360^{\circ}$ around this point source is obtained $[2,4,5]$. The specifications of the point source are as follows: length of the source $\leq 2 \mathrm{~mm}$, Inner diameter of the source $\leq 1.6 \mathrm{~mm}$ and activity $200 \mu \mathrm{Ci}$ of ${ }^{99 \mathrm{~m}} \mathrm{Tc}$.

For COR measuring, it was acquired a 360 degree tomographic study using a $64 \times 64$ matrix, 20\% window and 64 projection. Each projection requires $50 \mathrm{~K}$ counts acquisition $[4,8]$. COGX and COGY were calculated in each projection using relation 2 and 3 and offset error $R(\theta)$ as a function of rotation angle $(\theta)$ was calculated by relation 7 [4]. The COGY offset should be independent of angle so almost a linear plot should be obtained $[4,8]$.

$R(\theta)=[(N+1)-\operatorname{COGX}(\theta)-\operatorname{COGX}(\theta+180)] / 2$

Here, $\mathrm{N}($ matrix size $)=64$.

In consideration of the specific characteristics of AQCP, a new method has been introduced for COR assessment. In this new method, the camera is holding in a fixed position and AQCP moves the point source on the circle with radius R. In the other words, $\mathbf{A Q C P}$ rotates and positions the point source on the AQCP's center of rotation (cor) with the radius R (Fig. 2). For the assessment of COR in the AQCP method, axis of AQCP rotation should be in parallel with the axis of SPECT rotation. Offset error R_cor $(\theta)$ of AQCP method is calculated pursuant to the relation 7 same as what is done in the IAEA-TECDOC-602 method.

\section{RESULTS}

Uniformity Test: The planar extrinsic uniformity over the UFOV and the absorbed dose of technologist were measured in both the IAEA-TECDOC-602 and AQCP methods and the results are shown in Table 2 . The reproducibility of IU was evaluated by analyzing 10 extrinsic flood acquisitions with 2 million total counts. Uniformity check carried out in 2-3 min in IAEA-TECDOC-602 method and in 5-7 min in AQCP method.

The mean (+/- S.D.) of 10 measurements of IU parameter using IAEA-TECDOC-602 method is $9.56(+/-0.959)$ while by AQCP method it's $9.55(+/-0.92)$. The Maximum and the

Table 2. Quantitative Assessment of the Planar Extrinsic Uniformity and the Absorbed Dose of Worker with IAEA-TECDOC-602 and AQCP Methods

\begin{tabular}{|c|c|c|c|c|c|c|}
\hline 2 & 9 & 6.2 & 157 & 9 & 0.8 & 320 \\
\hline 3 & 10 & 6 & 162 & 10 & 1.0 & 416 \\
\hline 5 & 11.3 & 6.4 & 179 & 11.25 & 0.9 & 380 \\
\hline 6 & 9.1 & 5.9 & 142 & 9.1 & 0.8 & 327 \\
\hline 7 & 10.2 & 6 & 169 & 10.2 & 0.7 & 305 \\
\hline 8 & 8.7 & 6.1 & 170 & 8.7 & 0.8 & 330 \\
\hline
\end{tabular}


average error difference between AQCP and IAEATECDOC-602 methods for evaluating of IU parameter are $0.1 \%$ and $0.01 \%$.

The mean (+/- S.D.) of 10 measurements of the absorbed dose of technologist during the flood field uniformity in IAEA-TECDOC-602 method is 6.18(+/- 0.19) $\mu \mathrm{Sv}$ and the absorbed dose of technologist in AQCP method is $0.82(+/-$ $0.10) \mu \mathrm{Sv}$.

Collimator Hole Angulation (CHA) Test: In the CHA measurement, the results of the three collimators of LEHR, LEHS and LEUHR were examined and the results obtained shown in Fig. (3) and Table 3.

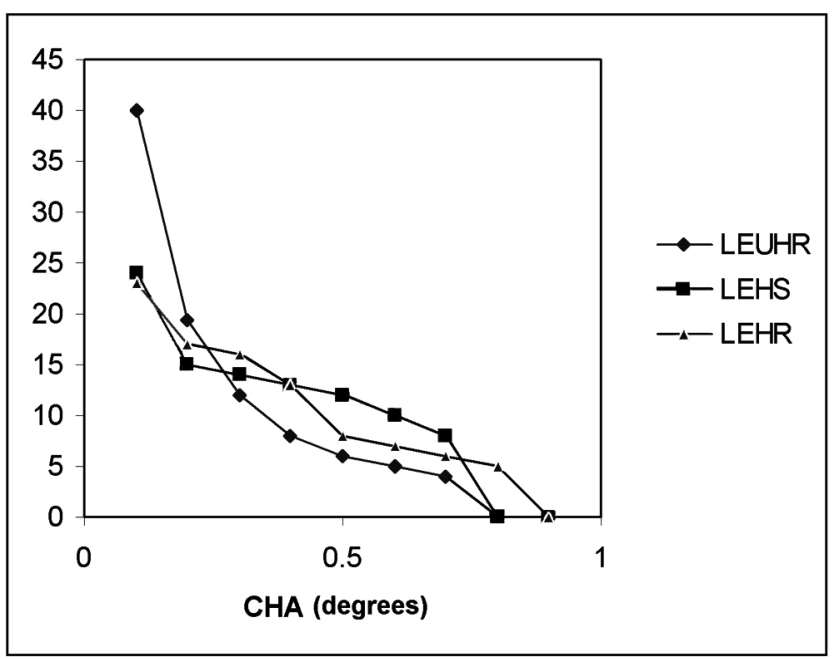

Fig. (3). Statistical changes in CHA for LEHR, LEHS and LEUHR collimators (Y Axis is percentage of pixels).

Repetition measurements for the three types of collimators including those obtained after re-running of the AQCP program gave an S.D. of less $0.1^{\circ}$ for each point of collimator. CHA test was conducted within an average time of 3035 min. The RMS (+/-S.D.) of CHA for 140 positions of LEHR, LEHS and LEUHR collimators were $0.290^{\circ}(+/-$ $\left.0.207^{\circ}\right), \quad 0.292^{\circ}\left(+/-0.197^{\circ}\right)$ and $0.208^{\circ}\left(+/-0.184^{\circ}\right)$ respectively. These are very similar to the results obtained in other relevant studies $[1,2]$.
The Maximum of CHA for LEHR, LEHS and LEUHR collimators were $0.78^{\circ}, 0.67^{\circ}$ and $0.66^{\circ}$ respectively.

In addition, it has been proved that the measured RMS of CHA for LEHR collimator with the distance variation from the collimator's surface $+/-1 \mathrm{~mm}$ has been varied only $+/-0.04$ degree. The results for LEHR collimator indicate that a $1 \mathrm{~mm}$ change in $(\mathrm{Z} 1-\mathrm{Z2})((\mathrm{Z} 1-\mathrm{Z} 2)=(31 \mathrm{~mm}))$ changing the RMS of $\mathrm{CHA}$ from $0.29^{\circ}$ to $0.33^{\circ}$ and $-1 \mathrm{~mm}$ change in (Z1-Z2) ((Z1$\mathrm{Z} 2)=(29 \mathrm{~mm})$ ) changing the RMS of CHA from $0.29^{\circ}$ to $0.25^{\circ}$.

It should be mentioned that CHA is dependent on the variation of the distance of the two images. For precise measurement of CHA, the precise distance of the point source movement should be measured vertically away from collimator face.

Center of Rotation Test: The results of measurement of COR offset $(R(\theta))$ in terms of SPECT rotation angle and the results of COR offset and error (R_cor $(\theta))$ in terms of AQCP rotation angle can be seen in Table $\mathbf{4}$. COR test was conducted within an average time $8-10 \mathrm{~min}$. with the two afore-said methods.

\section{DISCUSSION}

Uniformity Test: The differences in the IU measurement were primarily due to the Poisson statistics in the pixel data while all other factors are the same [15]. Investigations show that the most radiation exposures received by nuclear medicine technologist is due to flood phantom imaging comparing to any other nuclear medicine procedures [9]. The reported exposure during IAEA-TECDOC-602 flood uniformity is 0.58 $\mathrm{mR}(6.04 \mu \mathrm{Sv})$ and does not include exposure from preparation and administration of radiopharmaceutical [9] but in our measurement the dose preparation and administration was included. The systematic uniformity test's results show a considerable reduction of technologist dose compared to the IAEA-TECDOC-602 method due to the use of AQCP method. The reasons for the technologist dose reduction are as follows:

1- The lower activity of line source when compared to the activity of flood source,

2- The easier administration of the line source in terms of dose when comparing to the dose administration by the flood phantom,

Table 3. The Collimator Test Results

\begin{tabular}{|c|c|c|c|}
\hline Maximum of CHA (Degree) & Standard Deviation (Degree) & RMS of CHA (Degree) & Collimator \\
\hline \hline 0.78 & 0.207 & 0.290 & LEHR \\
\hline 0.67 & 0.197 & 0.292 & LEHS \\
\hline 0.66 & 0.184 & 0.209 & LEUHR \\
\hline
\end{tabular}

Table 4. The COR Offset Error of SPECT with IAEA-TECDOC-602 and AQCP Methods (All Measurements in Pixel Unit)

\begin{tabular}{|c|c|c|c|c|c|}
\hline $\begin{array}{c}\text { Deviation } \\
\text { of Y Offset }\end{array}$ & $\begin{array}{c}\text { Error Rang in } \\
\text { Y Axis }\end{array}$ & $\begin{array}{c}\text { Maximum and } \\
\text { Minimum Offset of } \\
\text { Y Axis }\end{array}$ & $\begin{array}{c}\text { Deviation of } \\
\text { X Offset }\end{array}$ & $\begin{array}{c}\text { Error Rang } \\
\text { in X Axis }\end{array}$ & $\begin{array}{c}\text { Maximum and Minimum } \\
\text { Offset of X Axis }\end{array}$ \\
\hline \hline 0.31 & 1.26 & $0.57,-0.69$ & 0.28 & 1.23 & $0.73,-0.49$ \\
\hline 0.293 & 1.13 & $0.52,-0.61$ & 0.269 & 0.94 & $0.53,-0.41$ \\
\hline
\end{tabular}


3- The automatic adjustment of line source with AQCP in comparison with the manual adjustment of flood phantom.

Collimator Hole Angulation (CHA) Test: The CHA test's results for the three collimators are very similar to the results [1,2]. Since AQCP can position the point source with absolute accuracy $0.018^{\circ}$, the CHA can therefore be measured more precisely and be interpreted visually. So it indicates that the precise measurement of the distance of the two images ((Z1-Z2) in relations 4 and 5) has a great effect on the measurement of CHA.

Center of Rotation test: The experiments were shown that there is a slight difference in the determination of COR offset and the standard deviation between the IAEATECDOC-602 method and AQCP method. By comparing the findings of this method with the previous one, it can be realized that the rotation of SPECT system can not be ideally précise. It can be seen in Table 4 that the standard deviation of COR in X and Y Axis in the Standard Method are 0.28 and 0.31 whilst in AQCP method the standard deviation of COR in X and Y Axis are 0.269 and 0.293. All these measurements have been conducted in Pixel Unit. These two parameters (COR offset and Standard Deviation) are varied due to mechanical problems with detector rotation, changes in amplifier gain and offset, problem with head tilt, collimator hole angulation, errors in the analog to digital converter, as well as the lack of parallelism between the collimator/detector plane and the axis of rotation [4]. In IAEATECDOC-602 method of COR evaluation, we measured and applied the constant shift of COR correction. Based on such comparison between the two afore described methods, the mechanical problems with detector rotation should be considered as the main cause of the difference between the two methods. Our findings show 7\% difference between maximum deviations of COR in two methods. Consequently, the authors could find out that by applying the correction of detector rotation for different angles of data acquisition, the image reconstructed from back projection of SPECT will not be blurred, and this would result in a higher resolution image.

\section{CONCLUSION}

AQCP is a computerized phantom and has other potential ability as a QC phantom. AQCP moves a radioactive source in the FOV of an imaging NM device on a definite path to produce any spatial distribution of gamma rays to simulate other QC phantoms. AQCP phantom has several hardware and software sections for establishing a three dimensional distribution of $\gamma$ rays by moving a source in the FOV of im- aging system. AQCP can optimize QC tests and QC program.

The advantages of AQCP in comparison with classic methods are: reduction of radioactive material consumption, reduction of radiation exposure to the staff, reduction of QC test cost, implementation of QC program with one phantom, perform a uniform set of procedures, increase the accuracy and precision of some QC tests, automation of the measurements and evaluation process make by AQCP suitable for both acceptance tests and routine quality control checks.

\section{ACKNOWLEDGEMENTS}

It is upon the authors of the present Paper to sincerely and cordially present their special thanks and appreciation to the Administration and the staff of the Karaj Nuclear Medicine and Agricultural Research Center and especially to Dr. Raisali, Dr. Karimian and Mrs. Moradkhani as well as to the National Radiation Protection Department of Iran.

\section{REFERENCES}

[1] Malmin RE, Stanley PC, Guth WR. Collimator Angulation Error and Its Effect on SPECT. J Nucl Med 1990; 31:655-659.

[2] Busemann-Sokole E. Measurement of Collimator Hole Angulation and camera head tilt for Slant and Parallel Hole collimators used in SPECT. J Nucl Med 1987; 28:1592-1598.

[3] Chang W, Li SQ, Williams JJ, et al. New methods of examining gamma camera collimators. J Nucl Med 1988; 29:674-683.

[4] Rotating Scintillation Camera SPECT Acceptance Testing and Quality Control. AAPM report No:22; American Association of Physicists in Medicine, College Park, MD. 1987.

[5] Quality Control of Nuclear Medicine Instruments. IAEA TECDOC 602, International Atomic Energy Agency, Vienna: Austria; 1991.

[6] Eckholt M, Bergmann H. Angulation errors in parallel-hole and fan beam collimators: computer controlled quality control and acceptance testing procedure. J Nucl Med 2000; 41:548-555.

[7] Takahashi Y, Murase K, Higashno H, et al. SPECT imaging with off-set detector system: comparison of sampling angles 2, 4 and 6 degrees. Ann Nucl Med 2002; 16:343-347.

[8] Hines H, Kayayan R, Colsher J, et al. Recommendations for implementing SPECT instrumentation quality control. Nuclear Medicine section-National Electrical Manufacturers Association (NEMA). Eur J Nucl Med 1999; 26:527-32.

[9] Dowd SB. Practical radiation protection and applied radiobiology. W. B. Saunders Co: Philadelphia; 1994.

[10] Smith EM. Scintillation camera Quality Control, part 1: Establishing the quality control program. J Nucl Med Technol 1998; 26:913 .

[11] Hackett MT, Magoun SL, Thompson RB. Nonuniformity Intrinsic flood artifact caused by point source syringe needle. J Nucl Med Technol 1997; 25: 41-43.

[12] Thomas GB. Calculus and Analytic Geometry. 4th ed. Addison Wesley Reading:MA; 1969.

[13] Kuo BC. Automatic control systems. Fifth ed. Upper Saddle River, NJ: Prentice-Hall; 1987.

[14] www.NI.com. web site of National Instruments Corporation.

[15] Young KC, Kouris K, Awdeh M, et al. Reproducibility and action levels for gamma camera uniformity. Nucl Med Commun 1990; 11:95-101. 